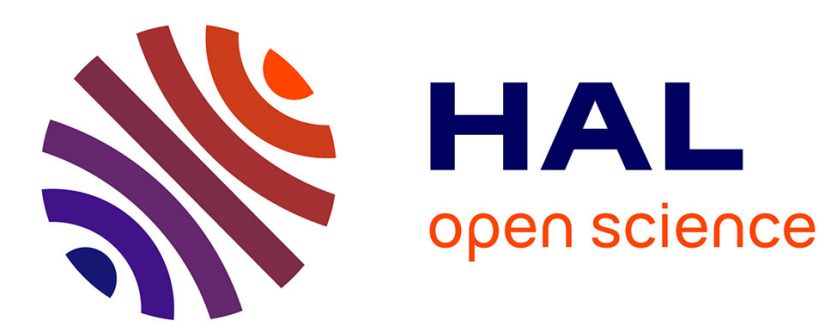

\title{
Etude, par diffusion des neutrons, de l'ordre local dans une phase micellaire ionique directe
}

\author{
C. Cabos, P. Delord, J.C. Martin
}

\section{To cite this version:}

C. Cabos, P. Delord, J.C. Martin. Etude, par diffusion des neutrons, de l'ordre local dans une phase micellaire ionique directe. Journal de Physique Lettres, 1979, 40 (16), pp.407-411. 10.1051/jphyslet:019790040016040700 . jpa-00231655

\section{HAL Id: jpa-00231655 https://hal.science/jpa-00231655}

Submitted on 1 Jan 1979

HAL is a multi-disciplinary open access archive for the deposit and dissemination of scientific research documents, whether they are published or not. The documents may come from teaching and research institutions in France or abroad, or from public or private research centers.
L'archive ouverte pluridisciplinaire HAL, est destinée au dépôt et à la diffusion de documents scientifiques de niveau recherche, publiés ou non, émanant des établissements d'enseignement et de recherche français ou étrangers, des laboratoires publics ou privés. 


\title{
Etude, par diffusion des neutrons, de l'ordre local dans une phase micellaire ionique directe
}

\author{
C. Cabos, P. Delord et J. C. Martin \\ Groupe de Dynamique des Phases Condensées (*), Laboratoire de Minéralogie Cristallographie, \\ Université des Sciences et Techniques du Languedoc, place Eugène-Bataillon, 34060 Montpellier Cedex, France
}

(Reçu le 31 janvier 1979, révisé le 15 juin 1979, accepté le 20 juin 1979)

\begin{abstract}
Résumé. - Nous avons étudié, par diffusion des neutrons, les corrélations intermicellaires dans des solutions aqueuses d'un tensioactif ionique.

Si on fait l'hypothèse que la taille et la forme des micelles varient peu avec la concentration, la formule de Zernicke et Prins permet de calculer les fonctions de distribution radiales, qui montrent que les solutions micellaires ioniques directes sont fortement corrélées.
\end{abstract}

\begin{abstract}
We have studied by small angle scattering of neutrons, intermicellar correlations in aqueous solutions of a ionic tensioactive substance.

If we suppose that the size and the shape of the micelles do not strongly change with the concentration, the Zernicke and Prins formula allows us to calculate radial distribution functions which show that the direct ionic micellar solutions are strongly correlated.
\end{abstract}

1. Introduction. - Le diagramme de diffusion neutronique aux petits angles, des solutions micellaires ioniques directes (têtes polaires de molécules d'amphiphiles dirigées vers l'extérieur de la micelle), présente la superposition de deux effets : à la diffusion individuelle des particules se superpose un phénomène de corrélations intermicellaires qui se manifeste par la présence d'un anneau.

Nous avons vérifié que cet anneau est caractéristique des micelles ioniques directes car on ne l'observe pas dans le cas de solutions micellaires inverses ou non ioniques. On peut, d'autre part, faire disparaître cet anneau de corrélation par adjonction de sels qui écrantent les interactions électrostatiques.

Nous avons pu, dans un travail précédent [1], déterminer les paramètres descriptifs de la micelle en utilisant la méthode du contraste variable, à condition d'étudier des solutions très diluées (2 à 3 fois la concentration micellaire critique).

L'objet de cette lettre est de décrire l'ordre local dans la phase micellaire et d'étudier sa variation en fonction de la concentration. Nous ferons l'hypothèse que, dans le domaine de concentrations étudié (c.m.c. $<c<0,5$ mole/1), toutes les micelles sont

(*) Laboratoire associé au C.N.R.S. sphériques et identiques. L'hypothèse de monodispersité d'une solution de concentration donnée est celle que nous avons faite précédemment $[1,2]$; elle est indispensable pour l'exploitation de l'information, mais il est clair que ce n'est qu'une première approximation. En effet, le spectre de diffusion aux petits angles d'une solution monodisperse de micelles sphériques devrait présenter des maximas successifs, ce que l'on n'observe dans aucun cas (quel que soit le contraste ou la concentration). Ceci s'explique par le fait qu'une faible polydispersité dans la taille des particules suffit pour brouiller le diagramme de diffusion qui ne présente alors qu'une décroissance monotone (Fig. 1).

La seconde hypothèse - invariance de la masse des micelles avec la concentration - n'a pas de valeur générale mais peut être faite dans le cas particulier de cette étude. Deux arguments peuvent en effet être avancés pour justifier ce point. D'une manière générale, Tanford [3] souligne que dans le domaine des petites micelles (nombre d'agrégation de l'ordre de 100) constituées d'amphiphiles à une chaîne paraffinique simple, seules des petites variations de tailles se produisent. Dans le cas du p-octylbenzènesulfonate de sodium, des expériences de biréfringence magnétique [4] ont permis de constater que ce tensioactif, à la différence d'amphiphiles tels que le $\mathrm{CTABr}$, 


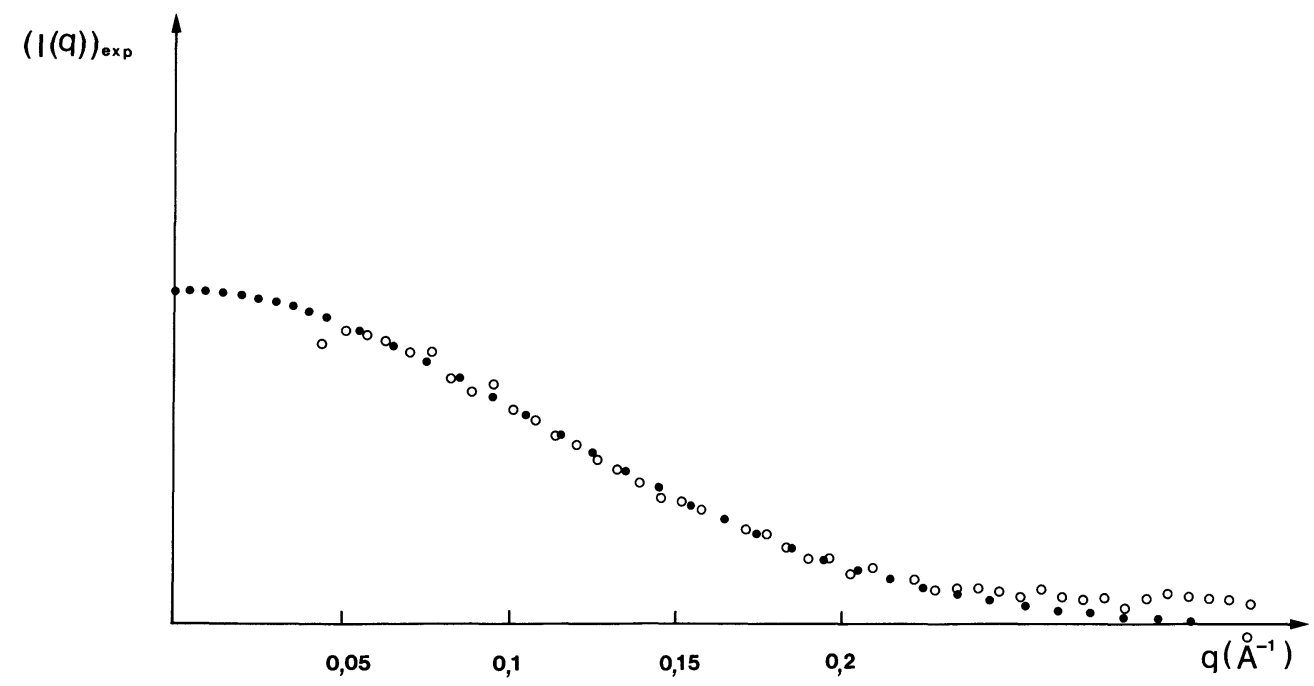

Fig. 1. - Détermination de $F^{2}(q)$. Les points $O$ représentent, à une échelle arbitraire, la variation de l'intensité diffusée par la solution la plus diluée $\left(c_{n}=0,03\right.$ mole $\left.1^{-1}\right)$. Les points $\bullet$ sont relatifs au graphe de la fonction $b \mathrm{e}^{a q^{*}}$. Les paramètres $a$ et $b$ sont calculés lorsque les deux courbes sont confondues dans la zone de Guinier. Le carré du facteur de structure de la micelle est ainsi donné, à un facteur constant près, par chaque point de l'exponentielle.

$\left[F^{2}(q)\right.$ determination. The open circles $O$ show, on an arbitrary scale, the intensity variation versus $q$ for the more dilute solution $\left(c_{0}=0.03\right.$ mole $\left.^{-1}\right)$. The full circles $\bullet$ are relative to the graph of the function $b \mathrm{e}^{a q^{2}}$. The $a$ and $b$ parameters are computed when the two graphs are in coincidence in the Guinier zone. The square of the structure factor is thus given by each value of the exponential function except for a constant factor.]

ne présente pas de transition vers de grosses micelles allongées.

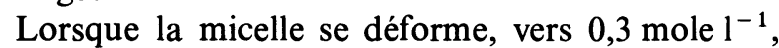
l'allongement observé ne dépasse pas $20 \%$. Cette déformation est suffisamment faible pour que notre hypothèse soit acceptable en première approximation.

On peut alors utiliser la formule classique de Zernicke et Prins [5] qui donne l'expression de l'intensité observable $I(q)$ du rayonnement diffusé par un ensemble de $N$ particules, contenues dans le volume $V$, en fonction de l'angle de diffusion $2 \theta$ :

$$
\begin{aligned}
I(q) & =N F^{2}(q) \times \\
\times & {\left[1-\frac{1}{v_{1}} \int_{0}^{\infty}\left[1-P\left(r, v_{1}\right)\right] \frac{\sin q r}{q r} 4 \Pi r^{2} \mathrm{~d} r\right] }
\end{aligned}
$$

où $q$ désigne le module du vecteur diffusion, $\lambda$ étant la longueur d'onde du rayonnement.

$F(q)$ est le facteur de structure de chaque particule. $v_{1}$ est le volume moyen offert à chaque micelle $\left(v_{1}=V / N\right)$.

La fonction de répartition $P\left(r, v_{1}\right)$, [6] permet de définir la probabilité $P$ de trouver à la fois le centre d'une particule dans un élément de volume $\mathrm{d} v_{\mathrm{k}}$ et le centre d'une autre particule dans le volume $\mathrm{d} v_{\mathrm{i}}$.

$$
P=\frac{\mathrm{d} v_{\mathrm{k}}}{v_{1}} \frac{\mathrm{d} v_{\mathrm{i}}}{v_{1}} P\left(r, v_{1}\right)
$$

Si on suppose qu'il n'y a pas d'ordre à grande distance, la quantité $[1-P(r)]$ tend vers zéro quand $r$ tend vers l'infini.
On peut envisager deux exploitations différentes de la relation (1). La première, développée notamment par Yvon [7] et Born et Green [8], utilisant la théorie statistique des fluides, conduit à une expression thermodynamique de $I(q)$ permettant, dans certains cas, de calculer le potentiel dont dépendent les forces interparticulaires.

La seconde, qui sera seule illustrée ici, permet d'obtenir une représentation géométrique simple de l'ordre local à l'échelle micellaire. En effet, sans hypothèses autres que celles qui ont été faites, on peut, pour une concentration donnée, calculer par transformée de Fourier la fonction de répartition et en déduire la fonction de distribution radiale

$$
1-P\left(r, v_{1}\right)=\frac{v_{1}}{2 \Pi^{2} r} \int_{0}^{\infty}\left[1-\frac{I(q)}{N F^{2}(q)}\right] q \sin q r \mathrm{~d} q
$$

$$
\begin{aligned}
4 \Pi r^{2} \frac{P\left(r, v_{1}\right)}{v_{1}} & =\frac{4 \Pi r^{2}}{v_{1}}+ \\
+\frac{2 r}{\Pi} & \int_{0}^{\infty} q\left[\frac{I(q)}{N F^{2}(q)}-1\right] \sin q r \mathrm{~d} q
\end{aligned}
$$

Cette dernière équation fournit la probabilité

$$
4 \Pi r^{2} \frac{P(r)}{v_{1}} \mathrm{~d} r
$$

de trouver le centre d'une micelle à une distance comprise entre $r$ et $r+\mathrm{d} r$ du centre d'une autre micelle. La courbe représentative de la fonction (3) 
permettra donc de calculer le nombre moyen de premières voisines d'une micelle donnée.

2. Etude expérimentale. - Les solutions étudiées sont des solutions micellaires de p.octylbenzènesulfonate de sodium. Cet amphiphile a été synthétisé au laboratoire suivant une technique déjà exposée et a fait l'objet d'études préliminaires [2] qui ont permis de déterminer sa concentration micellaire critique (c.m.c.). Par diffusion des neutrons aux petits angles (D.N.P.A.), nous avons déterminé [1] les caractéristiques de la micelle : rayon de giration, densité de longueur de diffusion cohérente de la partie polaire et masse molaire.

On dispose en D.N.P.A. d'un choix étendu de solvants, de l'eau protonée à l'eau totalement deutériée. C'est $\mathrm{D}_{2} \mathrm{O}$ qui a été retenue ici pour deux raisons : la première est qu'on réalise ainsi un contraste maximum entre la particule et le solvant, ce qui permet d'obtenir un signal élevé. La deuxième raison est que, dans ce cas, le carré du facteur de structure a une variation monotone. On peut le vérifier en examinant l'expression de $F(q)$ pour un modèle sphérique à deux niveaux de densité.

$$
\begin{aligned}
F(q)=\frac{4}{3} \Pi\left[R_{\mathrm{par}}^{3}\left(\rho_{\mathrm{par}}-\rho_{\mathrm{pol}}\right)\right. & \Phi\left(q R_{\mathrm{par}}\right)+ \\
& \left.+R_{\mathrm{pol}}^{3}\left(\rho_{\mathrm{pol}}-\rho_{\mathrm{S}}\right) \Phi\left(q R_{\mathrm{pol}}\right)\right]
\end{aligned}
$$

où $R_{\text {par }}$ et $R_{\text {pol }}$ sont les rayons des parties paraffiniques et polaires de la micelle, $\rho_{\mathrm{par}}$ et $\rho_{\mathrm{pol}}$ étant les densités de longueur de diffusion cohérentes correspondantes et $\rho_{\mathrm{S}}$ celle du solvant avec

$$
\Phi(q R)=3 \frac{\sin q r-q r \cos q r}{(q r)^{3}} .
$$

Si le solvant est $\mathrm{D}_{2} \mathrm{O}$, alors [1], $\rho_{\text {par }}<\rho_{\text {pol }}<\rho_{\mathrm{D}_{2} \mathrm{O}}$ et $F^{2}(q)$ reste une fonction décroissante de $q$. Par contre pour $\rho_{\text {par }}<\rho_{\mathrm{S}}<\rho_{\text {pol }} F^{2}(q)$ peut présenter un maximum [9] dans la même zone que l'anneau de corrélation. Des solutions micellaires dans $\mathrm{D}_{2} \mathrm{O}$ ont été préparées pour des concentrations croissantes de $c=0,03$ mole $/ 1$ jusqu'à $c=0,5$ mole $/ 1$. Cette étude a été réalisée sur le montage expérimental de diffusion des neutrons - D 17 - installé sur le réacteur à haut flux de l'Institut Laüe Langevin à Grenoble. Toutes les mesures ont été faites à $40^{\circ} \mathrm{C}$. La cellule utilisée est une cuve plane d'épaisseur $1 \mathrm{~mm}$. Le domaine exploré en $q$ étant assez étendu (jusqu'à $2 \theta=20^{\circ}$ pour $\lambda=7,0 \AA$, soit une résolution de $20 \AA$ ) il a été nécessaire d'effectuer des corrections de volume diffusant pour obtenir $I(q)$.

3. Traitement des résultats. - L'hypothèse de travail est que, dans le domaine de concentration étudié, le milieu reste monodisperse et que les dimensions micellaires sont constantes. Cette hypothèse qui entraîne l'invariance du facteur de structure permet un traitement mathématique simple du problème.

3.1 Détermination DE $F^{2}(q)$. - Le facteur de structure n'a pas été calculé au moyen de la formule (3) - la détermination de $R_{\mathrm{pol}}$ reste délicate [1] mais déduite de l'expérience. Nous avons fait l'hypothèse que l'intensité diffusée par la solution la plus diluée (Fig. 1) $\left(c_{0}=0,03\right.$ mole $/ 1$ soit trois fois la c.m.c. environ) dans le domaine où $I(q)$ suit la loi de Guinier, était proportionnelle à $F^{2}(q)$, ce qui revient à supprimer le terme d'interférence dans la relation (1).

Cependant, $F^{2}(q)$ doit être connu dans tout l'espace réciproque. Ceci a pu être obtenu par la détermination de la fonction exponentielle $b \mathrm{e}^{-a q^{2}}$ (où $a$ et $b$ sont des paramètres d'ajustement) dont le profil se superpose au mieux avec la courbe expérimentale dans la zone de Guinier [10] définie par la relation

$$
q R_{\mathrm{G}}<1,2 .
$$

On peut donc éçrire que pour cette concentration :

$$
b \mathrm{e}^{-a q^{2}} \sim N_{0,03} F^{2}(q)
$$

où $N_{0,03}$ représente le nombre de micelles contenues dans le volume diffusant $V$ constant dans nos expériences.

3.2 Mise À L'ÉCHELle DES COURBES EXPÉRIMENTALES. - La figure 2 représente les courbes de diffusion relatives à trois concentrations dont la plus faible $c_{1}$ est très grande devant $c_{0}\left(c_{1} \approx 10 c_{0}\right)$.

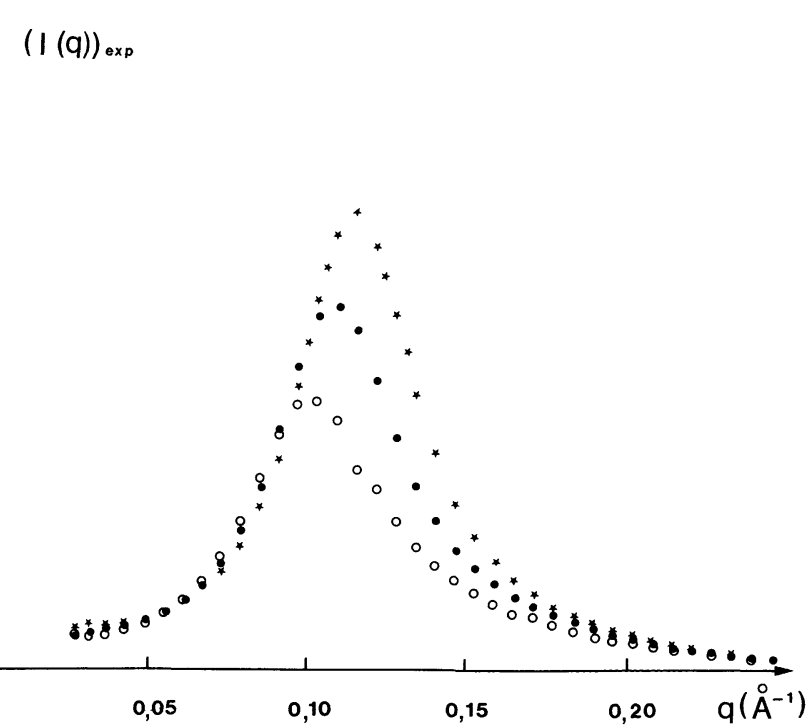

Fig. 2. - Variation de l'intensité diffusée (à une échelle arbitraire) pour trois concentrations : $\bigcirc c_{1}=0,30$ mole $1^{-1}$;

- $c_{2}=0,41$ mole $1^{-1} ; \forall c_{3}=0,52$ mole $1^{-1}$.

[Diffracted intensity, on an arbitrary scale, for three concentrations : $O c_{1}=0.30$ mole $^{-1}$;

- $c_{2}=0.41 \mathrm{~mole}^{-1} ; * c_{3}=0.52 \mathrm{~mole}^{-1}$.] 
Les effets de corrélations ne sont plus négligeables et se traduisent par un maximum, dont le déplacement vers les grandes valeurs de $q$ - pour des concentrations croissantes - est qualitativement significatif d'une diminution des distances intermicellaires.

Sur cette figure, les intensités notées $[I(q)]_{\text {exp }}$ sont données à une échelle arbitraire et ne peuvent être directement comparées à l'expression $I(q)$ de l'éq. (1). Il est donc nécessaire de définir une mise à l'échelle des valeurs expérimentales.

Pour une concentration $c$ quelconque et un nombre $N_{c}$ de micelles dans le volume $V$, on pourra calculer le rapport $\frac{I(q)}{N_{c} F^{2}(q)}$ par la relation :

$$
\frac{I(q)}{N_{c} F^{2}(q)}=\frac{[I(q)]_{\exp }}{b \mathrm{e}^{-a q^{2}}} \frac{N_{0,03}}{N_{c}}
$$$$
\text { avec } \quad \frac{N_{0,03}}{N_{c}}=\frac{0,03-\text { (c.m.c.) }}{c-\text { (c.m.c.) }} \text {. }
$$

On sait par ailleurs que pour les grandes valeurs de $q$, le rapport $I(q) / N_{c} F^{2}(q)$ tend vers 1 car les interférences intermicellaires deviennent négligeables. La

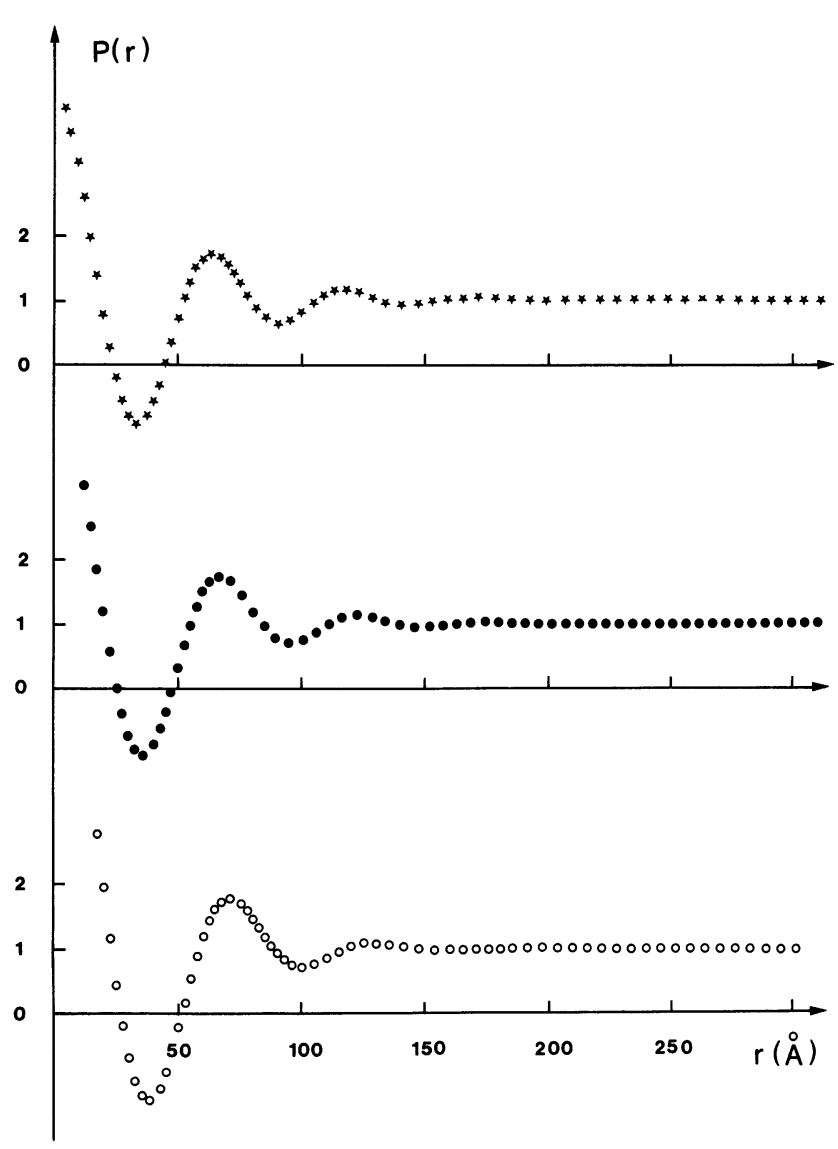

Fig. 3. - Fonction de répartition $P\left(r, v_{1}\right)$ relatives à trois concentrations : $O c_{1}=0,30$ mole $1^{-1}$ :

- $c_{2}=0,41$ mole $^{-1} ; * c_{3}=0,52$ mole $^{-1}$.

[Repartition functions $P\left(r, v_{1}\right)$ for three concentrations.

$O c_{1}=0.30 \mathrm{~mole}^{-1} ; \bullet c_{2}=0.41 \mathrm{~mole}^{-1}$;

$* c_{3}=0.52 \mathrm{~mole}^{-1}$.] méthode de normalisation précédente permet de préciser à partir de quelle valeur $q_{c}$ de $q$ cette limite est atteinte : pour toutes les concentrations étudiées $q_{c} \approx 0,15 \AA^{-1}$. Pour $q>q_{c}$, on prendra 1 comme valeur de $I(q) / N_{c} F^{2}(q)$, ce qui garantit la convergence des intégrales (2) et (3).

L'extrapolation de $[I(q)]_{\exp }$ au voisinage de $q=0$ ne pose pas de problèmes pratiques car dans ce domaine les valeurs de $I(q)$ ont une contribution négligeable au calcul de l'intégrale (2). On peut simplement remarquer [6], en se limitant à l'intensité observable exprimée par l'éq. (1) que $[I(0)]_{\exp }$ doit être de l'ordre de $\frac{N_{c}}{N_{0,03}} b$ pour les solutions diluées.

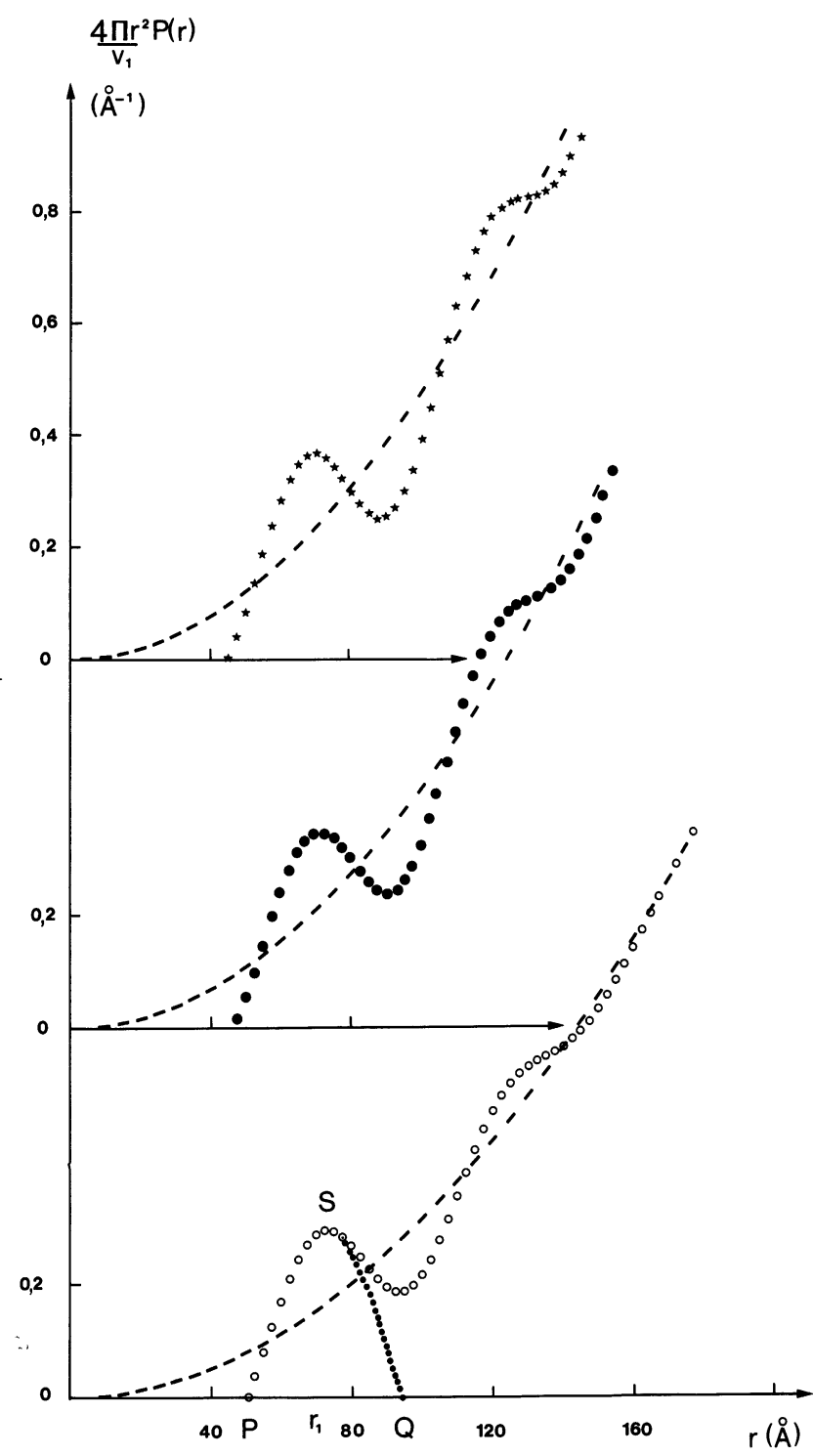

Fig. 4. - Fonctions de distribution radiale pour les trois concentractions étudiées : $O c_{1}=0,30$ mole $^{-1}$;

- $c_{2}=0,41 \mathrm{~mole}^{-1} ; * c_{3}=0,52 \mathrm{~mole}^{-1}$.

[Radial distribution functions for the three studied concentrations : $O c_{1}=0.30$ mole $^{-1}$;

- $c_{2}=0.41 \mathrm{~mole}^{-1} ; * c_{3}=0.52 \mathrm{~mole}^{-1}$.] 
3.3 FONCTIONS DE RÉPARTITION ET FONCTIONS DE DISTRIBUTION RADIALE. - La fonction $\frac{I(q)}{N F^{2}(q)}$ étant maintenant définie dans tout l'espace réciproque, les formules (2) et (3) permettent de calculer $P\left(r, v_{1}\right)$ et $4 \Pi r^{2} \frac{P(r)}{v_{1}} \cdot v_{1}$ est déterminé pour chaque valeur de la concentration à partir du nombre de monomères par micelle qui est [1] $n=82 \pm 3$. Pratiquement $v_{1}=13,6 \times 10^{4} / c\left(\AA^{3}\right)$.

Sur les courbes $P\left(r, v_{1}\right)$, on peut observer (Fig. 3) un premier maximum très net dont l'abscisse diminue légèrement lorsque la concentration augmente. Corrélativement, des maximums secondaires plus flous apparaissent et, pour la concentration la plus élevée, le troisième ordre est observable.

Les fonctions de distribution radiale représentées sur la figure 4 permettent de préciser, dans un domaine $r$ plus limité autour d'une particule placée à l'origine 0 , l'ordre local à l'échelle micellaire. Soit $r_{1}$ l'abscisse du premier pic $\mathrm{S}$ qui apparaît bien détaché du reste de la courbe. D'après la définition de $P\left(r, v_{1}\right)$, on peut dire que l'aire se trouvant sous le premier pic de la fonction $\frac{4 \Pi r^{2}}{v_{1}} P\left(r, v_{1}\right)$ représente le nombre $p$ de micelles dans la première sphère de coordination.

Nous avons choisi ici de définir le premier pic (Fig. 4) par le profil PSQ dont la partie SQ est symétrique de PS par rapport à SI, ce qui conduit à une valeur par défaut du nombre de coordination $p$. La résolution par cette méthode du deuxième pic paraît plus délicate car son abscisse est imprécise.
Le tableau suivant donne les valeurs de $r_{1}$ et de $p$ pour diverses concentrations.

Jusqu'à $c=0,3$ mole $1^{-1}$, qui indique la limite du domaine dans lequel notre hypothèse de travail paraît bien vérifiée [4], les valeurs de $p$ obtenues (avec une précision de $4 \%$ environ) suggèrent un ordre micellaire de type cubique centré.

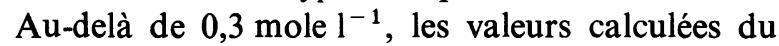
nombre de coordination présentent une incertitude plus importante. En effet, à un allongement des micelles de $20 \%$ correspond une variation du volume micellaire pouvant atteindre $50 \%$ dans l'hypothèse d'un modèle d'ellipsoïde allongé ou aplati. L'incertitude qui en résulte sur le terme $v_{1}$ n'affecte pratiquement pas la position du maximum de corrélation $\left(r_{1}\right)$ mais diminue la précision dans la détermination de $p$.

Tableau des résultats expérimentaux

$\begin{array}{ccc}\begin{array}{c}c \\ \left(\mathrm{~mole}^{-1}\right)\end{array} & r_{1} & p \\ - & - & - \\ 0,1 & 108 & 7,4 \pm 0,3 \\ 0,15 & 99 & 7,4 \pm 0,3 \\ 0,2 & 87 & 7,4 \pm 0,3 \\ 0,25 & 82 & 8,1 \pm 0,3 \\ 0,3 & 77 & 8,1 \pm 0,3 \\ 0,341 & 72 & 10 \pm 2 \\ 0,408 & 71 & 11 \pm 2 \\ 0,456 & 70 & 12 \pm 2 \\ 0,516 & 69 & 12 \pm 2\end{array}$

\section{Bibliographie}

[1] Cabos, C. et Delord, P., J. Physique 39 (1978) 432.

[2] Cabos, C., Delord, P. et Rouvière, J., J. Appl. Cryst. 10 (1977) 37.

[3] TANFORD, C., The hydrophobic effect : formation of micelles and biological membranes (New York, John Wiley) 1973.

[4] Porte, G. et Poggi, Y., Phys. Rev. Lett. 41 (1978) 1481.

[5] Zernicke, F. et Prins, J. A., Z. Phys. 41 (1927) 184.
[6] Fournet, G., Handbuch der Physik 32 (1957) 238.

[7] Yvon, J., Actual. Sci. (Paris, Hermann) 1937, p. 543.

[8] Born, M. et Green, H. S., Proc. R. Soc. A 188 (1946) 10.

[9] Reiss-Husson, F. et Luzzati, V., J. Phys. Chem. 68 (1964) 3504.

[10] Guinier, A. et Fournet, G., Small-Angle Scattering of $X$-Rays (New York, John Wiley) 1955. 